ISBN 978-93-84422-76-9

6th International Conference on Developments in Engineering and Technology

(ICDET-2017)

Bangkok (Thailand) Feb.6-7, 2017

\title{
Membrane Filtration of Pine oil Emulsion: Design and Application of a Novel Shear Enhanced Module
}

\author{
Mithu Naskar ${ }^{1}$, Kalpana Biswas ${ }^{1}$, Chiranjib Bhattacharjee ${ }^{2}$, Debasish Sarkar $^{1} *$ \\ ${ }^{1}$ Department of Chemical Engineering, University of Calcutta, Kolkata: 700009, India \\ ${ }^{2}$ Department of Chemical Engineering, Jadavpur University, Kolkata; 700032, India
}

\begin{abstract}
Oil-water emulsion is one of the hazardous of industrial effluent to be treated before discharge. Membrane filtration has been recognized as an effective remediation process for the treatment of oily wastewater. However, oil being a potent membrane fouling species, the filtration process often suffers the drawback of permeate flux decline because of operational nonidealities, such as concentration polarization and membrane fouling. Use of high membrane shear has long been identified as a suitable technique to overcome these nonidealities. The present work illustrates the design and performance characterization of a novel shear enhanced membrane module, named as Intermeshed Spinning Basket Membrane (ISBM) module. The module is unique in terms of its inherent hydrodynamic cleaning facility. The module consists of two intermeshed baskets, each with four rectangular arms. The alternate sides of the arms are fitted with flat sheet, Polyvinyledene Fluoride (PVDF) membrane of $50 \mathrm{kDa}$ molecular cut off. Pine oil-water emulsion with varying concentration has been chosen as the feed. In addition to the feed concentration, the effects of transmembrane pressure (TMP) and the rotational speed of basket on permeate flux were also explored. The maximum permeate flux was observed to be as high as $1.6543 \times 10^{-4} \mathrm{~m}^{3} \cdot \mathrm{m}^{-2} \cdot \mathrm{s}^{-1}$ at a TMP of $3.5 \mathrm{bar}$ and at a basket speed of $300 \mathrm{rpm}$ for a $3 \% \mathrm{v} / \mathrm{v}$ pine oil emulsion. Moreover, the reverse rotation induced hydrodynamic cleaning run enables the module to regenerate the steady state flux to 76-85\% of the corresponding start up value, even after a continuous operation for $9 \mathrm{~h}$.
\end{abstract}

Keywords: Intermeshed spinning Basket Membrane; Shear enhanced membrane module; permeate flux; cleaning run.

\section{Introduction}

Over the last two decades membrane based separation processes have emerged enormously. The domain of application includes food processing, water and waste water treatments, pharmaceutics, biotechnology, medical device and many others [1]. However, membrane based processes suffer from a severe drawback of massive flux decline with time. The well known phenomena of reversible concentration polarization and irreversible membrane fouling (which in turn is regarded as a consequence of the first) are primarily responsible for transient flux decline in general [2]. It is evident that to reduce the fouling led permanent flux decline, growth of the polarized solute layer on the membrane surface must be restricted to a minimum [3]. This is because once the flux decline is beyond a threshold, membrane cleaning is the only option left in order to partially recover the permeate flux. Although frequent membrane cleaning increases the operational cost and may damage the membrane material leading to a reduced service life [4].

Dynamic or shear enhanced (DSE) modules, first commercialized in late eighties, constitute a separate class of filtration units, which can generate a very high membrane shear without increasing the feed flow rate. A standard DSE module must consists of either rotating or a vibrating part. For example, in the first commercialized DSE module a cylindrical membrane rotates at a very high speed inside a concentric cylindrical casing [5]. Different 
types of DSE modules were developed later on, with a primary objective to generate high membrane shear rate as well as membrane surface area. DSE modules with high speed rotating disk(s) close to a flat stationary membrane or with rotating membranes are reported to produce higher permeate flux compared to the cross flow units of the same membrane surface area and power input [6,7]. However, all the DSE modules proposed so far, still suffers from the problem of transient flux decline, though the percentage reduction is much lower than a standard cross flow module. As a consequence, once the flux drops below a certain threshold, membrane cleaning is the only option, which can be accomplished either chemically or by hydrodynamically. In a sequel of recent articles [8-10], we have reported the design, hydrodynamic and performance characteristics of a new DSE module with self-cleaning facility.

The present work has been undertaken in an attempt to improve the design of SBM module in terms of higher membrane shear rate, specific membrane surface area and still better flux recovery. In the present module, two spinning baskets are intermeshed with a phase difference of $45^{\circ}$. Accordingly, the module has been named as intermeshed spinning basket membrane (ISBM) module. In the present study, the module has been characterized under different basket rotational speed $(\Omega)$, feed concentration $\left(C_{0}\right)$ and transmembrane pressure (TMP) in filtration of pine oil emulsion using Polyvinyledene Fluoride (PVDF) membrane of $50 \mathrm{kDa}$ molecular cut off.

\section{Experimental}

\subsection{ISBM pilot}

The module primarily comprises of two identical spinning baskets mounted on two parallel hollow shafts. Each basket consists of four radial arm fitted with flat sheet membrane (effective filtration area: $105 \times 55 \mathrm{~mm}^{2}$ ) on one side while the other side is impermeable. The baskets are intermeshed by two identical spur gears (with involute teeth) fitted on the parallel shafts. One of the shaft is connected to a three phase, 3 HP induction motor by a suitable belt-pulley drive. The corresponding basket rotates in the same direction as that of the motor while the other revolves in the opposite direction. The whole system with proper sealing arrangement is placed inside stainless steel housing. The detail of the module and the basket assembly is shown in fig. 1.

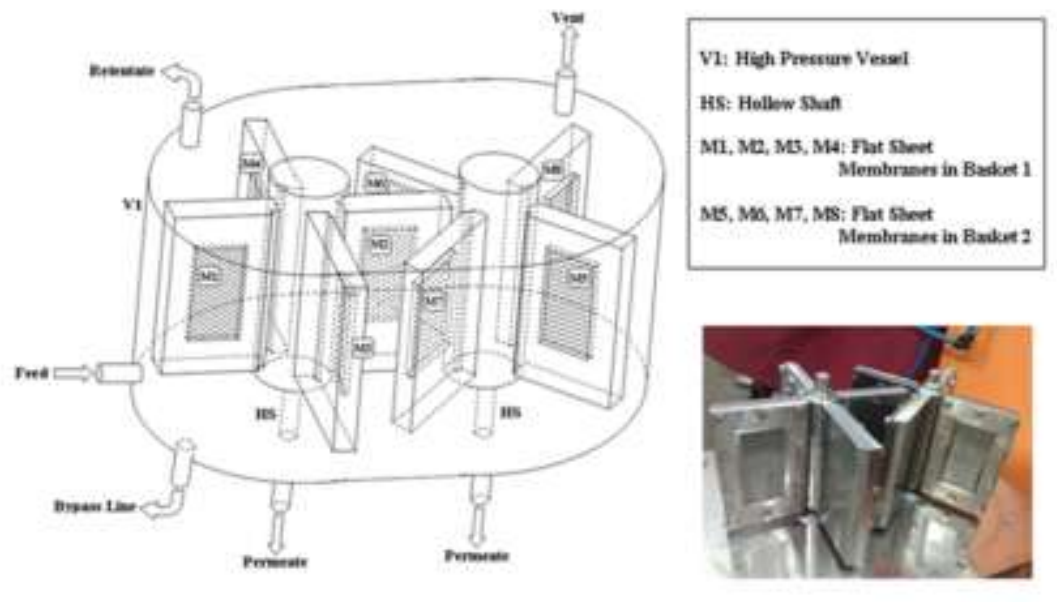

Fig. 1 Schematic diagram and photograph of ISBM module

During a normal run, the baskets are subjected to rotate at equal speed (in the opposite direction of course) in the directions of their respective membrane area vectors. This leads to a high membrane shear rate and consequently a high flux. However, like all DSE modules, the proposed ISBM module is also prone to flux decline. To initiate the subsequent cleaning run, the back pressure regulator has to be fully opened in order to release the TMP and baskets are rotated in the reverse direction (in the opposite direction of the membrane area vector). Because of reverse directional rotation, a local vacuum is expected to set in over the membrane surface. 
Accordingly, a back pressure (from permeate to retentate side) will come into play, which will displace the accumulated solute on the membrane surface, which reduces the hydrodynamic resistance of the membrane and thereby recover the permeate flux in the next normal run.

The present ISBM pilot, solely designed by us, was fabricated by EMCEE, Howrah, West Bengal (India) as the prescribed design. The induction motor, used in the pilot is fitted with a variable speed drive (ABB IP20/UL open type), which can also revert the direction of rotation.

\subsection{Experimental Procedure}

Initially, the module was pressurized with distilled water at a transmembrane pressure of $6 \mathrm{~kg} . \mathrm{cm}^{-2}$, which is higher than the highest operational pressure. The membrane compaction was continued for $4 \mathrm{~h}$ until the water flux attained a steady state value, which ensures the complete compaction of the membrane. The performance of the proposed module was studied with respect to three different process parameters, which are (1) applied TMP (2) rotational speed of the basket $(\Omega)$ and $(3)$ feed concentration $\left(\mathrm{C}_{0}\right)$. There retentate flow rate was held constant $(=$ $\left.1.33 \times 10^{-4} \mathrm{~m}^{3} . \mathrm{s}^{-1}\right)$ for all the experimental runs. Once the permeate flux attains a steady value and remains unchanged over a prolong interval, the module was switched to cleaning.

Each normal run was conducted for $9 \mathrm{~h}$. On the other hand, every cleaning run was conducted arbitrarily for 300 $\mathrm{s}$, as the run time duration is impossible to optimize a priori. Every experimental run consists of three successive normal runs, intervened by two cleaning runs. The same procedure was repeated for each experiment with a fixed set TMP, $\Omega$ and $\mathrm{C}_{0}$ values. The experiments were carried out varying the parameters in the following range: TMP $\left(\mathrm{kg} . \mathrm{cm}^{-2}\right) \Rightarrow[2.5,4], \Omega(\mathrm{rpm}) \Rightarrow[100,350]$ and $\mathrm{C}_{0}(\mathrm{v} / \mathrm{v} \%) \Rightarrow[3,6]$.

\subsection{Particle size analysis of the oil-water emulsion}

To prepare the pine oil-water emulsion, predicted amounts of pine oil and water were mixed along with tween-80 (surfactant) and the mixture was vigorously agitated for 5 minutes. After the initial mixing, the emulsion was added to the measured amount of water. This was treated as the feed solution. Same procedure was repeated to obtain the oil-water emulsion with different concentration.

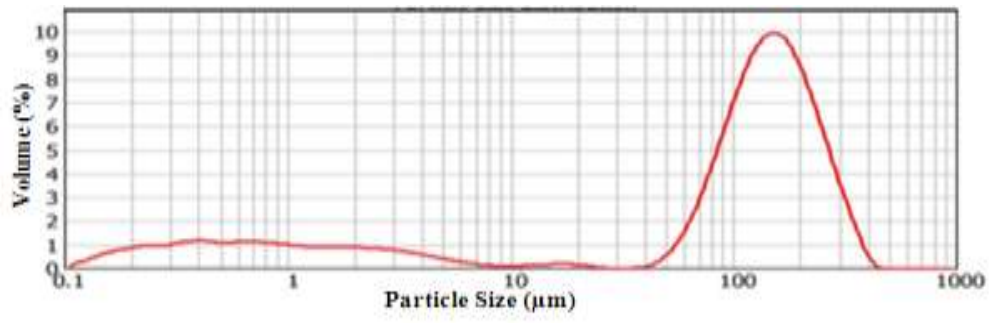

Fig. 2 Particle size distribution of pine oil in water emulsion

For the particle size characterization of the oil water emulsion, $50 \mathrm{ml}$ of the pine water oil emulsion was taken and the particle size was measured in Malvern equipment (Mastersizer 2000) by taking the reference refractive index of coconut oil. The particle size distribution, as obtained from the experiment, is shown in Fig. 2. The size was recorded to vary in the range of $56 \mu \mathrm{m}$ to $400 \mu \mathrm{m}$.

\section{Results and Discussion}

\subsection{Variation of the permeate flux with time at different transmembrane pressure}

To obtain the trend of the transient flux profile with the applied TMP, the rotational speed and the feed concentration were held constant at $200 \mathrm{rpm}$ and 3\% (v/v) respectively. The profiles are depicted in Fig. 3 (a). The transient flux was recorded to decrease with time. For example, at TMP of $4 \mathrm{~kg} . \mathrm{cm}^{-2}$, permeate flux was observed to decrease from $9.84 \mathrm{~m}^{3} \cdot \mathrm{m}^{-2} \cdot \mathrm{s}^{-1}$ to $8.64 \mathrm{~m}^{3} \cdot \mathrm{m}^{-2} \cdot \mathrm{s}^{-1}$ in the first normal run spanned from $\mathrm{t}=0 \mathrm{~min}$ to $\mathrm{t}$ $=180 \mathrm{~min}$, which alternately indicate a decrease of nearly 13\%. For other TMPs, the average decline of permeate flux was found to be around $20 \%$. However, it is worth noting that in cross flow systems, the decline from the start up to the steady state is reported to be more than 50\% [11]. Therefore, the results of the present study clearly justify the efficacy of the ISBM module in reducing the stated decline of the permeate flux. 
Additionally, as expected, both the steady and the transient flux were observed to increase monotonically with the applied transmembrane pressure. For example, as the TMP is increased from $2.5 \mathrm{~kg} . \mathrm{cm}^{-2}$ to $4 \mathrm{~kg} . \mathrm{cm}^{-2}$, the steady state permeate flux at the end of the first normal run i.e. at $\mathrm{t}=180 \mathrm{~min}$ was recorded to increase by $152 \%$. This may be simply explained from the well known osmotic pressure model, which predicts the permeate flux (J) to be related to TMP as

$$
J=\frac{T M P-\sigma \Delta \pi}{\mu R_{m}}
$$

Where $\Delta \pi=\pi\left(C_{m}\right)-\pi\left(C_{p}\right)$ is the osmotic pressure differential across the membrane, $\mu$ and $\mathrm{R}_{\mathrm{m}}$ representing the viscosity of the solution and hydraulic resistance of the membrane. Eq. 1 indicates that as TMP increases, the flux should increase monotonically. Nevertheless, the trend is not exactly proportional to TMP because of the term $\sigma \Delta \pi$. With the increase of TMP as the solute rejection increases, $\Delta \pi$ also increase according to the appropriate constitutive relation between the osmotic pressure and concentration. Accordingly, increased TMP causes a non-proportional increase of flux because of osmotic pressure effect. On the other hand, Fig. 3 (a-c) clearly depicts that after the first cleaning run, conducted only for $5 \mathrm{~min}$, the permeate flux was partially recovered to a value that is comparable to its start-up. For example, in case of the experimental run conducted at the TMP of $4 \mathrm{~kg} \cdot \mathrm{cm}^{-2}$, the regenerated permeate flux after the first cleaning run was noted to be $94 \%$ of its respective initial flux value, whereas the steady state flux before the first cleaning run was only $66 \%$. This alternatively means a flux enhancement nearly by $40 \%$ was achieved in the first cleaning run. Similar trends were noted for experimental runs. Moreover, even in the second cleaning run, an average regeneration more than $32 \%$ was observed. Therefore, the present module can be unambiguously confirmed to be a DSE module with efficient self-cleaning mechanism. The design is evident to serve the membrane industry with better performance particularly in case of high fouling feed solutions.
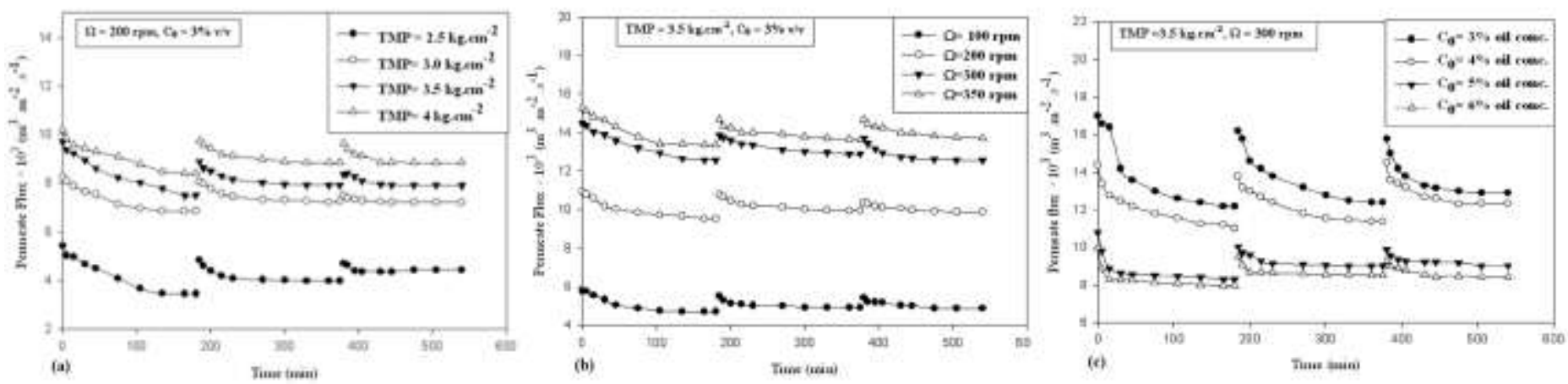

Fig. 3 Variation of permeate flux with time at different (a) transmembrane pressure (b) rotational speed and (c) feed concentration

\subsection{Variation of the permeate flux with time at different rotational speed}

Fig. 3 (b) depicts the variation of the permeate flux profiles with the rotational speed of baskets under constant TMP and $\mathrm{C}_{0}$. The figure represents an increasing trend of permeate flux with $\Omega$. For example, as the $\Omega$ was increased from 100 to $350 \mathrm{rpm}$, the steady state permeate flux at the end of the first normal run was recorded to increase by $133 \%$. Similar trend was observed at different other time intervals. The reason behind flux enhancement with $\Omega$ is purely due to reduced degree of polarization at higher $\Omega$. With the increase of rotational speed, the shear rate at the membrane surface rapidly increases, which removes the adsorbed solid and thereby reduces the mass transfer resistance. Additionally, high-speed rotation causes even more shear rate over the intermeshed region of the baskets. Therefore, the percentage flux enhancement with $\Omega$ is still higher compared to the Spinning Basket Membrane (SBM) module. In a SBM modules, for a similar change of $\Omega$, the steady state permeate, flux was reported to increase only by $83 \%$ in UF of bovine serum albumin [8-10].

\subsection{Variation of the permeate flux with time at different feed concentration}

The trend with feed concentration is represented in Fig. 3 (c). The figure shows that with the increase of $\mathrm{C}_{0}$, the permeate flux undergoes severe decline. This is evident because of enhanced polarization effect at higher 
concentration. Contrary to the membrane separation of standard solutions like BSA-water, PVA-water etc oilwater emulsion is different. In case of oil-water emulsion, oil droplets have a tendency to coalesce at the membrane surface and thereby to form a thin impermeable layer, which alternatively increases the osmotic pressure differential by many folds. Because of this, severe flux decline was observed with the increase of $\mathrm{C}_{0}$. With the increase of $\mathrm{C}_{0}$ from $3 \%$ to $6 \%(\mathrm{v} / \mathrm{v})$, the steady state permeate flux at the end of the first normal run was noted to decrease by $36 \%$. In case of BSA ultrafiltration, on the other hand, for a similar change of $\mathrm{C}_{0}$, the flux decline was only between $14 \%$ to $17 \%$. However, the cleaning run seems to be very efficient as after the first cleaning run, about $30 \%$ flux enhancement was achieved for all the experimental runs with different $\mathrm{C} 0$.

It may be noted that for all the experimental runs, the permeate samples were tested to check the presence of pine oil with the same particle-size analyzer. However, no trace of oil droplet was found, which confirms absolute rejection of the dispersed phase by the present PVDF membrane.

\section{Conclusion}

The design and performance characteristics of a self-cleaning enable dynamic shear enhanced module have been reported in the present article. The module is a modification of its baseline version, which is known as Spinning Basket Module. As in the present module, two spinning baskets are intermeshed together; it has been named as Intermeshed Spinning Basket Membrane (ISBM) module. The results indicate that the present ISBM module is able to restrict the permeate flux decline within $15 \%$ of its start up value after 9 hours of continuous operation. The maximum permeate flux was observed to be as high as $1.6 \times 10^{-2} \mathrm{~m}^{3} \cdot \mathrm{m}^{-2} \cdot \mathrm{s}^{-1}$ in separation of pine oil-water emulsion using a PVDF membrane. Therefore, the newly designed module may be confirmed to perform in a much superior way compared to the existing DSE modules particularly in treatment of high fouling feed streams.

\section{Acknowledgements}

The Financial assistance, provided by Department of Science and Technology (DST), Govt. of India, under Women Scientist Scheme (Ref. No.: SR/WOS-A/ET-89/2016 dated 17/11/2016) is gratefully acknowledged.

\section{Reference}

[1] Z. Zhang, Q. An, T. Liu, Y. Zhou, J. Qian and C. Gao, "Fabrication and characterization of novel $\mathrm{SiO}_{2}-\mathrm{PAMPS} / \mathrm{PSF}$ hybrid ultrafiltration membrane with high water flux," Desalination, Vol. 297, pp. 59-71, 2012. https://doi.org/10.1016/j.desal.2012.04.017

[2] G. Belfort, R. H. Davis and A. L. Zydney, "The behavior of suspensions and macromolecular solutions in crossflow microfiltration,” Journal of Membrane Science, Vol. 96, pp. 1-58, November 1994. https://doi.org/10.1016/0376-7388(94)00119-7

[3] G. Crozes, C. Anselme and J. Mallevialle, "Effect of adsorption of organic matter on fouling of ultrafiltration membranes," Journal of Membrane Science, Vol. 84, pp. 61-77, September 1993. https://doi.org/10.1016/0376-7388(93)85051-W

[4] D. A. Ladner, D. R. Vardon and M. M. Clark, "Effects of shear on microfiltration and ultrafiltration fouling by marine bloom-forming algae,” Journal of Membrane Science, Vol. 356, pp. 33-43, July 2010. https://doi.org/10.1016/j.memsci.2010.03.024

[5] S.S. Lee, B.G. Russotti and B. Buckland, "Microfiltration of recombinant yeast cells using a rotating disk dynamic filtration system," Biotechnology and Bioengineering, Vol. 48, pp. 386-400, November 1995. https://doi.org/10.1002/bit.260480411

[6] N. Hilal, O. O. Ogunbiyi, N. J. Miles and R. Nigmatullin, "Methods Employed for Control of Fouling in MF and UF Membranes: A Comprehensive Review," Separation Science and Technology, Vol. 40, pp. 1957-2005, April 2005. https://doi.org/10.1081/SS-200068409

[7] M. Y. Jaffrin, "Dynamic shear-enhanced membrane filtration: A review of rotating disks, rotating membranes and vibrating systems," Journal of Membrane Science, Vol. 324, pp. 7-25, October 2008.

https://doi.org/10.1016/j.memsci.2008.06.050 
[8] A. Sarkar, D. Sarkar, M. Gupta and C. Bhattacharjee, "Recovery of Polyvinyl Alcohol from Desizing Wastewater Using a Novel High-Shear Ultrafiltration Module," Clean - Soil Air Water, Vol. 40, pp. 830-837, August 2012. https://doi.org/10.1002/clen.201100527

[9] D. Sarkar, A. Sarkar, A. Roy and C. Bhattacharjee, "Performance characterization and design evaluation of spinning basket membrane (SBM) module using computational fluid dynamics (CFD)," Separation and Purification Technology ,Vol. 94, pp. 23-33, June 2012. https://doi.org/10.1016/j.seppur.2012.03.034

[10] A. Sarkar, S. Moulik, D. Sarkar, A. Roy and C. Bhattacharjee, "Performance characterization and CFD analysis of a novel shear enhanced membrane module in ultrafiltration of Bovine Serum Albumin (BSA)," Desalination, Vol. 292, pp. 53-63, April 2012. https://doi.org/10.1016/j.desal.2012.02.009 